ESAIM: PROCEEDINGS, November 2005, Vol.15, 75-86

T. Goudon, E. Sonnendrucker \& D. Talay, Editors

DOI: $10.1051 /$ proc:2005023

\title{
NUMERICAL SCHEMES FOR SOLVING THE NON-STATIONARY BOLTZMANN-POISSON SYSTEM FOR TWO-DIMENSIONAL SEMICONDUCTOR DEVICES *
}

\author{
Ch. Auer ${ }^{1}$, A. Majorana ${ }^{2}$ And F. Schürrer ${ }^{1}$
}

\begin{abstract}
In this paper we present a new way of discretizing the Boltzmann-Poisson system describing the electron transport in semiconductor devices. The new method is based on a cell average technique for the momentum space combined with a non-uniform discretization in real space. Results of the simulation of a 2D silicon MOSFET are shown and compared with Monte Carlo data. We found that the proposed scheme reduces both the number of grid points and the CPU time, without loss of accuracy.
\end{abstract}

Résumé. Dans cet article, on présente une nouvelle façon de discrétiser le système de BoltzmannPoisson décrivant le transport des électrons dans les semi-conducteurs. Cette nouvelle méthode est fondée sur des techniques de moyennes par cellule pour l'espace des moments combinées avec une discrétisation non uniforme sur l'espace réel. Des résultats de simulation d'un MOSFET 2D Silicone sont présentés et comparés avec des données Monte-Carlo. On trouve que le schéma proposé réduit à la fois le nombre de points de grille et le temps CPU, sans perte de précision.

\section{INTRODUCTION}

Very large scale integration is the forthcoming design in semiconductor technology. This implies that in modern electron devices the scale length of individual components becomes comparable with the distance between successive carrier interactions with the crystal. Consequently, the well-established drift-diffusion models describing the carrier transport lose their accuracy [1]. In highly integrated devices a consistent description of the dynamics of carriers is essential for a deeper understanding of the transport properties. The semiconductor Boltzmann transport equation (BTE) coupled with the Poisson equation provides a general theoretical framework for modeling the semiclassical electron transport in such devices [2].

Deterministic as well as stochastic procedures can be considered as solution approaches to these extremely sophisticated equations. So far, mainly stochastic methods have been applied to solve the BTEs [3]- [6]. Compared to stochastic methods, deterministic approaches offer noise-free resolution, high accuracy and easiness of arbitrary moment evaluations at low computational cost. Recently, Carrillo et. al. [7,8] succeeded in introducing a deterministic high-order finite difference Weighted Essentially Non Oscillatory (WENO) solver for the

\footnotetext{
* Supported by the Fond zur Förderung der wissenschaftlichen Forschung, Vienna, under contract number P17438-N08, and by the European community programme IHP, contract number HPRN-CT-2002-00282 on behalf of the CNR, and by M.U.R.S.T. Cofin 2004 Mathematical problem of Kinetic Theories

${ }^{1}$ Institute of Theoretical and Computational Physics, Graz University of Technology, Graz, Austria;

e-mail: auer@itp.tu-graz.ac.at \& schuerrer@itp.tu-graz.ac.at

2 Dipartimento di Matematica e Informatica, Università di Catania, Catania, Italy; e-mail: majorana@dmi.unict.it
} 
solution of the one-dimensional Boltzmann-Poisson system for semiconductor devices. Moreover, they extended their numerical technique to cope with spatially two-dimensional geometries [9]. However, the treatment of two-dimensional problems requires a large number of grid points (a few million), which leads to a great amount of CPU time for each simulation.

In this paper, a cell average technique combined with a WENO scheme is presented for solving the coupled Boltzmann-Poisson system. This new numerical scheme is based on a finite-volume method for treating the dependence of the electron distribution function on the three-dimensional wave vector. A fifth-order WENO solver $[8,9]$ and its extension to two-dimensional non-uniform grids is applied for dealing with the spatial dependence of the distribution function. The resulting transport equations are used for simulating the charge transport in a silicon MOSFET.

\section{The Boltzmann-Poisson system}

The temporal evolution of the electron distribution function $f(\mathrm{t}, \mathbf{x}, \mathbf{k})$ in semiconductors depending on time $\mathbf{t}$, position $\mathbf{x}$ and electron wave vector $\mathbf{k}$ is governed by the Boltzmann transport equation [10]

$$
\frac{\partial f}{\partial \mathrm{t}}+\frac{1}{\hbar} \nabla_{\mathbf{k}} \varepsilon \cdot \nabla_{\mathbf{x}} f-\frac{q}{\hbar} \mathbf{E} \cdot \nabla_{\mathbf{k}} f=Q(f)
$$

where $\hbar$ is the reduced Planck constant, and $q$ denotes the positive elementary charge. The function $\varepsilon(\mathbf{k})$ is the energy of the considered conduction band measured from the band minimum. According to the Kane dispersion relation, $\varepsilon$ is the positive root of

$$
\varepsilon(1+\alpha \varepsilon)=\frac{\hbar^{2} k^{2}}{2 m^{*}}
$$

where $\alpha$ denotes the non-parabolicity factor and $m^{*}$ the effective electron mass. The Poisson equation

$$
\nabla_{\mathbf{x}}\left[\epsilon_{r}(\mathbf{x}) \nabla_{\mathbf{x}} V\right]=\frac{q}{\epsilon_{0}}\left[n(\mathrm{t}, \mathbf{x})-N_{D}(\mathbf{x})\right]
$$

for the electric potential $V$ couples the electric field $\mathbf{E}=-\nabla_{\mathbf{x}} V$ with the doping density $N_{D}$ and the electron density $n$, which equals the zero-order moment of the electron distribution function $f$. In equation $(3)$, $\epsilon_{0}$ is the vacuum dielectric constant and $\epsilon_{r}(\mathbf{x})$ labels the relative dielectric function depending on the considered material. The collision operator $Q(f)$ on the right-hand side of the transport equation (1) takes into account the electron-phonon interaction in terms of acoustic deformation potential and optical intervalley scattering [11]. For low electron densities, it reads

$$
Q(f)(\mathrm{t}, \mathbf{x}, \mathbf{k})=\int_{\mathbb{R}^{3}}\left[S\left(\mathbf{k}^{\prime}, \mathbf{k}\right) f\left(\mathrm{t}, \mathbf{x}, \mathbf{k}^{\prime}\right)-S\left(\mathbf{k}, \mathbf{k}^{\prime}\right) f(\mathrm{t}, \mathbf{x}, \mathbf{k})\right] d \mathbf{k}^{\prime}
$$

with the scattering kernel

$$
\begin{aligned}
S\left(\mathbf{k}, \mathbf{k}^{\prime}\right)= & \left(n_{q}+1\right) K \delta\left(\varepsilon\left(\mathbf{k}^{\prime}\right)-\varepsilon(\mathbf{k})+\hbar \omega_{p}\right)+n_{q} K \delta\left(\varepsilon\left(\mathbf{k}^{\prime}\right)-\varepsilon(\mathbf{k})-\hbar \omega_{p}\right) \\
& +K_{0} \delta\left(\varepsilon\left(\mathbf{k}^{\prime}\right)-\varepsilon(\mathbf{k})\right)
\end{aligned}
$$

where $K$ and $K_{0}$ are constant for silicon. The symbol $\delta$ indicates the Dirac distribution and $\omega_{p}$ is the constant phonon frequency. The occupation number of phonons in equilibrium at the fixed lattice temperature $T_{L}$ is given by

with the Boltzmann constant $k_{B}$.

$$
n_{q}=\left[\exp \left(\frac{\hbar \omega_{p}}{k_{B} T_{L}}\right)-1\right]^{-1}
$$


For the numerical treatment of the system (1) and (3), it is convenient to introduce suitable dimensionless parameters and variables. We assume $T_{L}=300 \mathrm{~K}$. Typical values for length, time and voltage are $\ell_{*}=10^{-6} \mathrm{~m}$, $t_{*}=10^{-12} s$ and $V_{*}=1$ Volt, respectively. Thus, we define the dimensionless variables

$$
(x, y, z)=\frac{\mathbf{x}}{\ell_{*}}, \quad t=\frac{\mathrm{t}}{t_{*}}, \quad \Psi=\frac{V}{V_{*}}, \quad\left(E_{x}, E_{y}, E_{z}\right)=\frac{\mathbf{E}}{E_{*}}
$$

with $E_{*}=0.1 V_{*} \ell_{*}^{-1}$ and

$$
E_{x}=-c_{v} \frac{\partial \Psi}{\partial x}, \quad E_{y}=-c_{v} \frac{\partial \Psi}{\partial y}, \quad c_{v}=\frac{V_{*}}{\ell_{*} E_{*}}
$$

In correspondence to [12] and [8], we perform a coordinate transformation for $\mathbf{k}$ according to

$$
\mathbf{k}=\frac{\sqrt{2 m^{*} k_{B} T_{L}}}{\hbar} \sqrt{w\left(1+\alpha_{K} w\right)}\left(\mu, \sqrt{1-\mu^{2}} \cos \varphi, \sqrt{1-\mu^{2}} \sin \varphi\right)
$$

where the new independent variables are the dimensionless energy $w=\frac{\varepsilon}{k_{B} T_{L}}$, the cosine of the polar angle $\mu$ and the azimuth angle $\varphi$ with $\alpha_{K}=k_{B} T_{L} \alpha$. The main advantage of the generalized spherical coordinates (6) is the easy treatment of the Dirac delta distribution in the kernel (5) of the collision term. In fact, the change of the variables (6) enables us to transform the integral operator (4) with the not regular kernel $S$ into an integral-difference operator, as shown in the following.

We are interested in studying two-dimensional problems in real space but, of course, in the whole threedimensional $\mathbf{k}$-space. It is useful to consider the new unknown function $\Phi$ related to the electron distribution function via

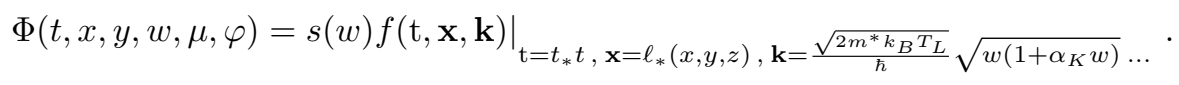

The function

$$
s(w)=\sqrt{w\left(1+\alpha_{K} w\right)}\left(1+2 \alpha_{K} w\right)
$$

is proportional to the Jacobian of the variable transformation (6) and, apart from a dimensional constant factor, to the density of states. This allows us to write the free streaming operator of the dimensionless Boltzmann equation in a conservative form, which is appropriate for applying standard numerical schemes used for hyperbolic partial differential equations. Due to the symmetry of the problem and of the collision operator, we have

$$
\Phi(t, x, y, w, \mu, 2 \pi-\varphi)=\Phi(t, x, y, w, \mu, \varphi)
$$

Straightforward but cumbersome calculations end in the following transport equation for $\Phi$ :

$$
\frac{\partial \Phi}{\partial t}+\frac{\partial}{\partial x}\left(g_{1} \Phi\right)+\frac{\partial}{\partial y}\left(g_{2} \Phi\right)+\frac{\partial}{\partial w}\left(g_{3} \Phi\right)+\frac{\partial}{\partial \mu}\left(g_{4} \Phi\right)+\frac{\partial}{\partial \varphi}\left(g_{5} \Phi\right)=C(\Phi)
$$


The functions $g_{i}(i=1,2, . ., 5)$ in the advection terms depend on the variables $w, \mu, \varphi$ as well as on time and position via the electric field:

$$
\begin{aligned}
g_{1}(\cdot) & =c_{x} \frac{\mu \sqrt{w\left(1+\alpha_{K} w\right)}}{1+2 \alpha_{K} w}, \\
g_{2}(\cdot) & =c_{x} \frac{\sqrt{1-\mu^{2}} \sqrt{w\left(1+\alpha_{K} w\right)} \cos \varphi}{1+2 \alpha_{K} w} \\
g_{3}(\cdot) & =-2 c_{k} \frac{\sqrt{w\left(1+\alpha_{K} w\right)}}{1+2 \alpha_{K} w}\left[\mu E_{x}(t, x, y)+\sqrt{1-\mu^{2}} \cos \varphi E_{y}(t, x, y)\right], \\
g_{4}(\cdot) & =-c_{k} \frac{\sqrt{1-\mu^{2}}}{\sqrt{w\left(1+\alpha_{K} w\right)}}\left[\sqrt{1-\mu^{2}} E_{x}(t, x, y)-\mu \cos \varphi E_{y}(t, x, y)\right], \\
g_{5}(\cdot) & =c_{k} \frac{\sin \varphi}{\sqrt{w\left(1+\alpha_{K} w\right)} \sqrt{1-\mu^{2}}} E_{y}(t, x, y)
\end{aligned}
$$

with

The right hand side of (9) is the integral-difference operator

$$
c_{x}=\frac{t_{*}}{\ell_{*}} \sqrt{\frac{2 k_{B} T_{L}}{m^{*}}} \text { and } c_{k}=\frac{t_{*} q E_{*}}{\sqrt{2 m^{*} k_{B} T_{L}}} .
$$

$$
\begin{aligned}
& C(\Phi)(t, x, y, w, \mu, \varphi)=s(w)\left\{c_{0} \int_{0}^{\pi} d \varphi^{\prime} \int_{-1}^{1} d \mu^{\prime} \Phi\left(t, x, y, w, \mu^{\prime}, \varphi^{\prime}\right)\right. \\
& \left.+\int_{0}^{\pi} d \varphi^{\prime} \int_{-1}^{1} d \mu^{\prime}\left[c_{+} \Phi\left(t, x, y, w+\gamma, \mu^{\prime}, \varphi^{\prime}\right)+c_{-} \Phi\left(t, x, y, w-\gamma, \mu^{\prime}, \varphi^{\prime}\right)\right]\right\} \\
& -2 \pi\left[c_{0} s(w)+c_{+} s(w-\gamma)+c_{-} s(w+\gamma)\right] \Phi(t, x, y, w, \mu, \varphi),
\end{aligned}
$$

where

$$
\left(c_{0}, c_{+}, c_{-}\right)=\frac{2 m^{*} t_{*}}{\hbar^{3}} \sqrt{2 m^{*} k_{B} T_{L}}\left(K_{0},\left(n_{q}+1\right) K, n_{q} K\right), \quad \gamma=\frac{\hbar \omega_{p}}{k_{B} T_{L}}
$$

are dimensionless parameters. We remark that the delta distributions in the kernel $S$ have been eliminated, which leads to the shifted arguments of $\Phi$. The parameter $\gamma$ represents the jump constant corresponding to the quantum of energy $\hbar \omega_{p}$. We have also taken into account the symmetry relation (8) for the integration with respect to $\varphi^{\prime}$.

In terms of the new variables the electron density becomes

$$
n\left(t_{*} t, \ell_{*} x, \ell_{*} y\right)=\int_{\mathbb{R}^{3}} f\left(t_{*} t, \ell_{*} x, \ell_{*} y, \mathbf{k}\right) d \mathbf{k}=\left(\frac{\sqrt{2 m^{*} k_{B} T_{L}}}{\hbar}\right)^{3} \rho(t, x, y),
$$

where

$$
\rho(t, x, y)=\int_{0}^{+\infty} d w \int_{-1}^{1} d \mu \int_{0}^{\pi} d \varphi \Phi(t, x, y, w, \mu, \varphi) .
$$

Hence, the dimensionless Poisson equation writes

$$
\frac{\partial}{\partial x}\left(\epsilon_{r} \frac{\partial \Psi}{\partial x}\right)+\frac{\partial}{\partial y}\left(\epsilon_{r} \frac{\partial \Psi}{\partial y}\right)=c_{p}\left[\rho(t, x, t)-\mathcal{N}_{D}(x, y)\right]
$$

with

$$
\mathcal{N}_{D}(x, y)=\left(\frac{\sqrt{2 m^{*} k_{B} T_{L}}}{\hbar}\right)^{-3} N_{D}\left(\ell_{*} x, \ell_{*} y\right) \text { and } c_{p}=\left(\frac{\sqrt{2 m^{*} k_{B} T_{L}}}{\hbar}\right)^{3} \frac{\ell_{*}^{2} q}{\epsilon_{0}}
$$


Choosing the same values of the physical parameters as in [12], we obtain

\begin{tabular}{|l|l|l|}
\hline$c_{0} \approx 0.2653$ & $c_{x} \approx 0.16857$ & $c_{p} \approx 0.183 \times 10^{7}$ \\
$c_{+} \approx 0.507$ & $c_{k} \approx 0.326067$ & $c_{v}=10$. \\
$c_{-} \approx 0.0443$ & & \\
\hline
\end{tabular}

\section{Numerical Scheme}

For obtaining approximate solutions to the coupled Boltzmann-Poisson system, we proceed as follows. In the first step we fix a maximum value $w_{\max }$ for the dimensionless energy. Of course, $w_{\max }$ must be related to the studied physical situation, and we must check that $\Phi(t, x, y, w, \mu, \varphi)$ with $w \geq w_{\max }$ is negligible for all $t$, $x, y, \mu$ and $\varphi$.

Next, we choose three suitable integer numbers $N_{w}, N_{\mu}$ and $N_{\varphi}$ and discretize the independent variables $w$, $\mu$ and $\varphi$ via

$$
\begin{array}{lll}
w_{k+\frac{1}{2}}=k \Delta w, & k=0,1, \ldots, N_{w}, & \Delta w=w_{\max } / N_{w}, \\
\mu_{m+\frac{1}{2}}=-1+m \Delta \mu, & m=0,1, \ldots, N_{\mu}, & \Delta \mu=2 / N_{\mu}, \\
\varphi_{n+\frac{1}{2}}=n \Delta \varphi, & n=0,1, \ldots, N_{\varphi}, & \Delta \varphi=\pi / N_{\varphi} .
\end{array}
$$

Here, we have taken into account that $\varphi \in[0, \pi]$. It is important to remark that $N_{w}$ must be chosen in such a way that $\sigma=\gamma / \Delta w \in \mathbb{N}$ in order to treat the shifted arguments in the collision operator accurately. We denote the generic cell in the $(w, \mu, \varphi)$ domain by

$$
\mathcal{Z}_{k m n}=\left[w_{k-\frac{1}{2}}, w_{k+\frac{1}{2}}\right] \times\left[\mu_{m-\frac{1}{2}}, \mu_{m+\frac{1}{2}}\right] \times\left[\varphi_{n-\frac{1}{2}}, \varphi_{n+\frac{1}{2}}\right]
$$

for $k=1,2, \ldots, N_{w}, m=1,2, \ldots, N_{\mu}$ and $n=1,2, \ldots, N_{\varphi}$. The center of the cell $\mathcal{Z}_{k m n}$ has the coordinates $\left(w_{k}, \mu_{m}, \varphi_{n}\right)$

Let $p(w, \mu, \varphi)$ be an assigned non-negative weight function. We consider the representation

$$
\Phi(t, x, y, w, \mu, \varphi)=p(w, \mu, \varphi) G(t, x, y, w, \mu, \varphi)
$$

of the distribution function with the new unknown $G$. Our main assumption is now that

$$
G(t, x, y, w, \mu, \varphi) \approx G\left(t, x, y, w_{k}, \mu_{m}, \varphi_{n}\right)=G_{k, m, n}(t, x, y)
$$

holds for every $t, x, y$ and $(w, \mu, \varphi)$ belonging to interior of the cell $\mathcal{Z}_{k m n}$. Concerning the derivation of the set of $N_{w} \times N_{\mu} \times N_{\varphi}$ evolution equations for the cell averages $G_{k, m, n}$ we refer to [13]. The treatment of this set of equations requires methods suitable for hyperbolic equations in conservative form. These schemes must be accurate also in the presence of strong gradients. The WENO scheme has given excellent results in solving Eqs. (1) and (3). Hence, we continue to use this method for treating the partial derivative with respect to $x$ and $y$. This technique gives a final set of ordinary differential equations in time, which are integrated by using TVD Runge-Kutta formulas [14]. The resulting scheme was tested by investigating the carrier transport in bulk silicon, in a $n^{+}-n-n^{+}$diode and in a silicon MESFET [13]. Comparisons of the results with those of a full WENO solver $[8,9]$ exhibited perfect agreement.

\section{Non-uniform Discretization in Real Space}

The schematic illustration of the considered MOSFET presented in figure 1 shows that the interfaces between high- and low-doping regions as well as the interface between the $\mathrm{Si}$ and the $\mathrm{SiO}_{2}$ regions are located in the upper half of the device. For this reason, the electron distribution function and the electric potential show steep gradients with respect to $x$ and $y$ in the upper half of the device, while in the lower half only small variations 


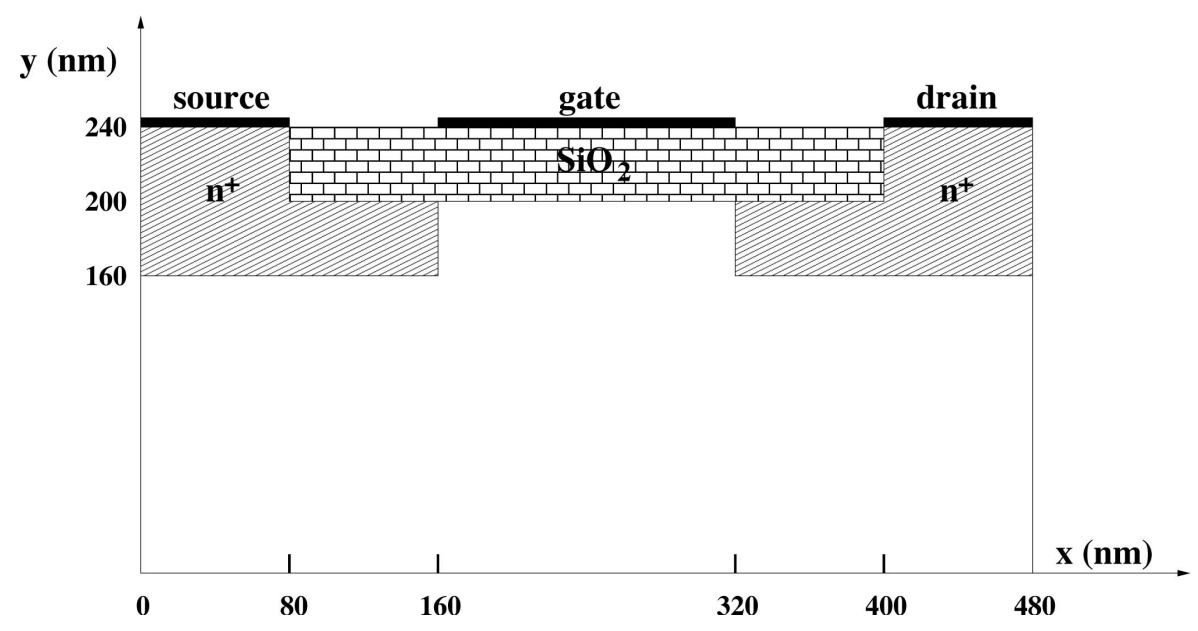

FIGURE 1. Schematic illustration of a 2D-MOSFET.

arise. This structure implies the application of a non-uniform space discretization in order to perform the computation in an efficient way. Different approaches for treating hyperbolic conservation laws on non-uniform meshes have been proposed in the literature and have been used for adaptive mesh refinement algorithms [15,16]. Most of the methods are formulated as second-order finite-volume schemes. In [17] a multidomain WENO finitedifference method has been presented. This approach, however, is not conservative at the interfaces between the different domains. Encouraged by the very good results obtained with the high-order WENO finite-difference scheme $[13,18]$ on uniform grids, we develop a strategy to apply this method to non-uniform discretizations in a conservative way.

First, we introduce two uniform rectangular discretizations

$$
\begin{aligned}
\mathcal{D} & =\left\{\left(x_{i}, y_{j}\right) \mid x_{i}=i \Delta x, y_{j}=j \Delta y, i=0, \ldots, N_{x}, j=0, \ldots, N_{y}\right\} \\
\mathcal{D}^{1} & =\left\{\left(x_{i}^{1}, y_{j}^{1}\right) \mid x_{i}^{1}=i \frac{\Delta x}{r}, y_{j}=y_{0}^{1}+j \frac{\Delta y}{r}, i=0, \ldots, N_{x}^{1}, j=0, \ldots, N_{y}^{1}\right\}
\end{aligned}
$$

in the $(x, y)$-domain. The parameters $\Delta_{x}, \Delta_{y}, N_{x}$ and $N_{y}$ are chosen in such a way that the grid $\mathcal{D}$ covers the entire device. In equation (14), the odd integer $r>1$ is the refinement factor. If we set

$$
N_{x}^{1}=r N_{x}, \quad N_{y}^{1}=r\left(N_{y}-j_{*}\right)-\frac{r+1}{2}, \quad y_{0}^{1}=\left(j_{*}+\frac{r+1}{2 r}\right) \Delta y,
$$

the grid $\mathcal{D}^{1}$ splits the considered domain in real space into two parts with an interface extended along the $\mathrm{x}$ direction and located at $\left(j_{*}+1 / 2\right) \Delta y$. Further, all grid points $\left(x_{i}, y_{j}\right)$ with $j>j_{*}$ of the coarse grid $\mathcal{D}$ coincide with certain positions on the fine grid $\mathcal{D}^{1}$. This especially holds for the boundary points $\left(0, y_{j}\right),\left(N_{x} \Delta x, y_{j}\right)$ with $j>j_{*}$ and $\left(x_{i}, N_{y} \Delta y\right)$ for $i=0, \ldots, N_{x}$.

Figure 2 shows the two grids at the interface for $r=3$. The grid points of the coarse grid $\mathcal{D}$ are marked by open squares $(\square)$, while the cross signs $(\times)$ display the positions of the grid points belonging to the fine mesh $\mathcal{D}^{1}$. Our method to solve the system of partial differential equations for $G_{k, m, n}$ on the non-uniform grid is based on WENO approximations $[8,9]$ of the derivatives $\partial_{x} G_{k, m, n}$ and $\partial_{y} G_{k, m, n}$ on the uniform grids $\mathcal{D}$ and $\mathcal{D}^{1}$. The conservative treatment of the interface between the fine and the coarse grid is realized by using a special update procedure for the numerical flux of the coarse grid at the interface. 


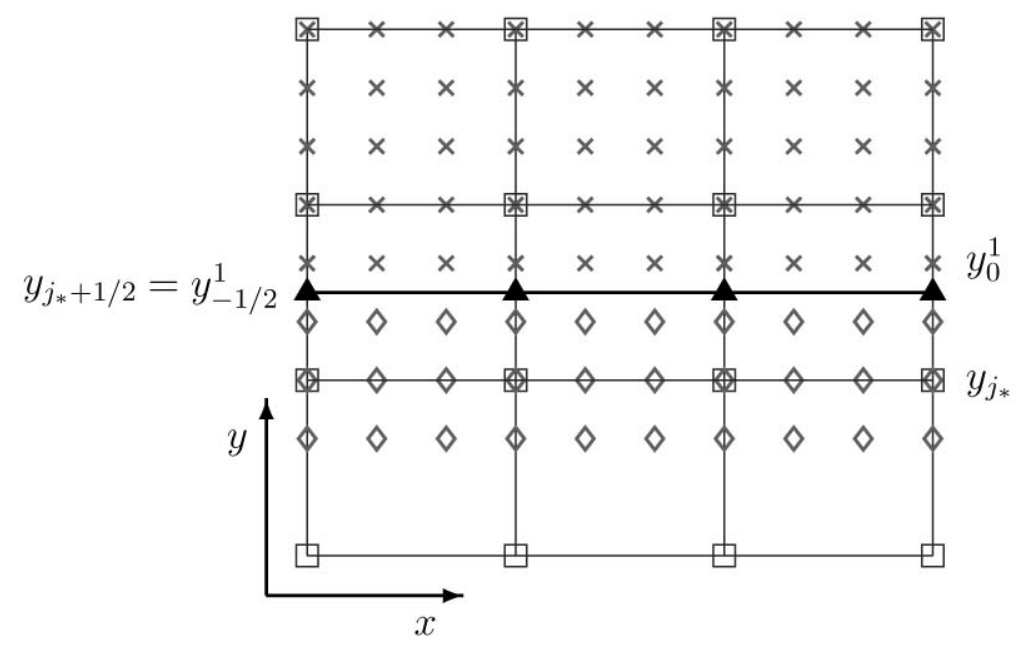

FiguRE 2. Interface of the non-uniform grid in real space.

At a certain time level $t_{0}$ we suppose that the quantities $G_{i, j, k, m, n}\left(t_{0}\right)=G_{k, m, n}\left(t_{0}, x_{i}, y_{j}\right)$ on the coarse grid and $G_{i, j, k, m, n}^{1}\left(t_{0}\right)=G_{k, m, n}\left(t_{0}, x_{i}^{1}, y_{j}^{1}\right)$ on the fine grid are known. Since the coarse mesh covers the entire $(x, y)$ domain, we can use the WENO scheme $[8,9]$ supplied by a suitable treatment of the boundaries to approximate the derivatives by

$$
\begin{aligned}
\partial_{x} G_{i, j, k, m, n}\left(t_{0}\right) & \approx\left[\hat{G}_{i+1 / 2, j, k, m, n}\left(t_{0}\right)-\hat{G}_{i-1 / 2, j, k, m, n}\left(t_{0}\right)\right] / \Delta x \\
\partial_{y} G_{i, j, k, m, n}\left(t_{0}\right) & \approx\left[\hat{G}_{i, j+1 / 2, k, m, n}\left(t_{0}\right)-\hat{G}_{i, j-1 / 2, k, m, n}\left(t_{0}\right)\right] / \Delta y
\end{aligned}
$$

Here, $\hat{G}_{i+1 / 2, j, k, m, n}\left(t_{0}\right)$ and $\hat{G}_{i, j+1 / 2, k, m, n}\left(t_{0}\right)$ represent the numerical fluxes with respect to the $x$ - and $y$ direction. Hence, we can obtain the solution $G_{i, j, k, m, n}\left(t_{1}\right)$ at time $t_{1}=t_{0}+\Delta t$ in all points of the coarse grid.

For the application of the fifth-order WENO approximation [8] to the derivatives $\partial_{y} G_{i, j, k, m, n}^{1}\left(t_{0}\right)$, it is necessary to know the distribution function at the $y$-positions $y_{-j}^{1}=y_{0}-j \Delta y / r$ for $j=1,2,3$. In Figure 2 these points are marked by the open diamonds $\diamond$. It should be further mentioned that due to the smaller discretization lengths the CFL-condition evaluated on the fine grid may force also smaller time increments $\Delta t^{1}$ compared to those of the coarse grid. Hence, we consider $\Delta t^{1}=\Delta t / r_{t}$ with the integer $r_{t} \geq 1$. For this reason, the quantities $G_{i,-j, k, m, n}^{1}\left(t_{0}+q \Delta t^{1}\right)$ for $q=0, \ldots, r_{t}-1$ are required to perform the time integration up to $t_{1}$ on the fine grid $\mathcal{D}^{1}$. We use a fifth-order centered Lagrange interpolation [17] to determine $G_{i,-j, k, m, n}^{1}\left(t_{0}\right)$ and $G_{i,-j, k, m, n}^{1}\left(t_{1}\right)$ from the known values $G_{i, j, k, m, n}\left(t_{0}\right)$ and $G_{i, j, k, m, n}\left(t_{1}\right)$ on the coarse grid. The desired solutions at the ghost points

$$
G_{i,-j, k, m, n}^{1}\left(t_{0}+q \Delta t^{1}\right)=\frac{r_{t}-q}{r_{t}} G_{i,-j, k, m, n}^{1}\left(t_{0}\right)+\frac{q}{r_{t}} G_{i,-j, k, m, n}^{1}\left(t_{1}\right)
$$

are calculated according to a linear interpolation with respect to time. At this point we are able to carry out the time integration on the fine grid $\mathcal{D}^{1}$ and obtain $G_{i, j, k, m, n}^{1}\left(t_{1}\right)$. Finally, $G_{i, j, k, m, n}\left(t_{1}\right)$ is replaced by the more accurate approximation $G_{i, j, k, m, n}^{1}\left(t_{1}\right)$ at all common points of $\mathcal{D}$ and $\mathcal{D}^{1}$.

Due to our construction of the non-uniform space discretization, we obtain

$$
y_{j_{*}+1 / 2}=y_{j_{*}}+\frac{\Delta y}{2}=y_{0}^{1}-\frac{\Delta y}{2 r}=y_{-1 / 2}^{1}
$$



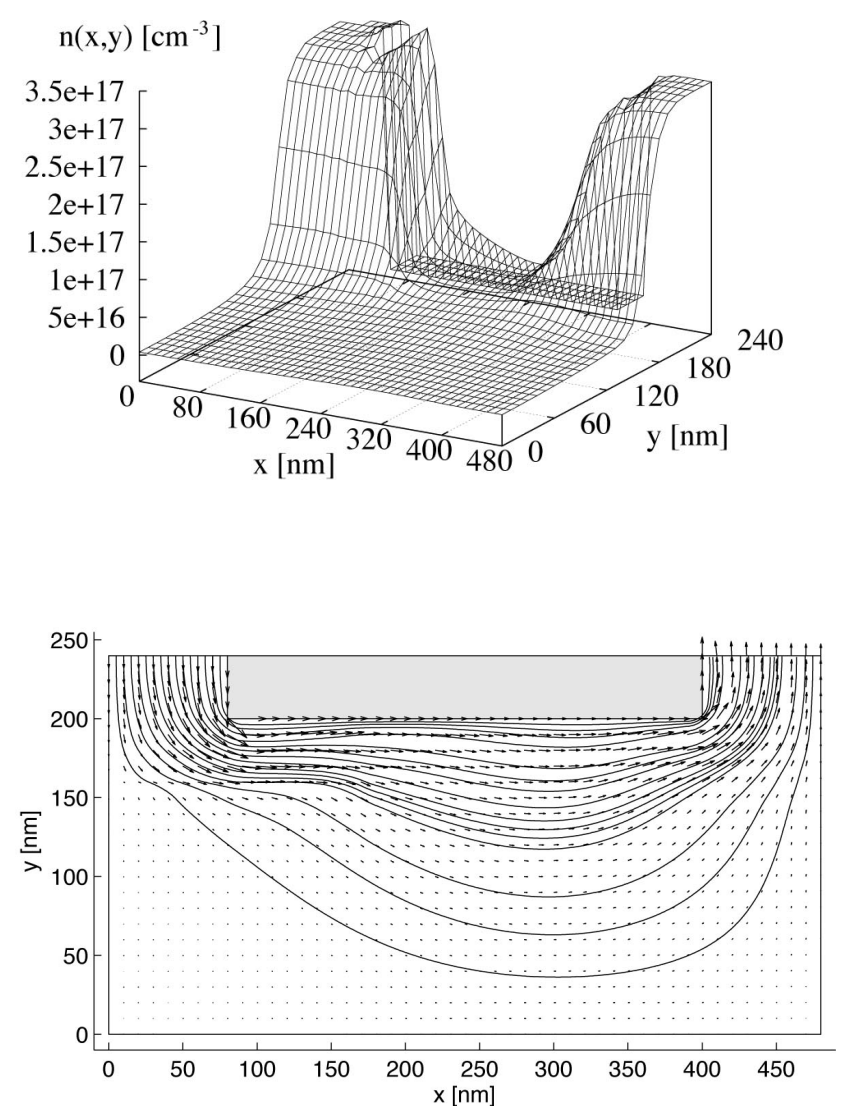
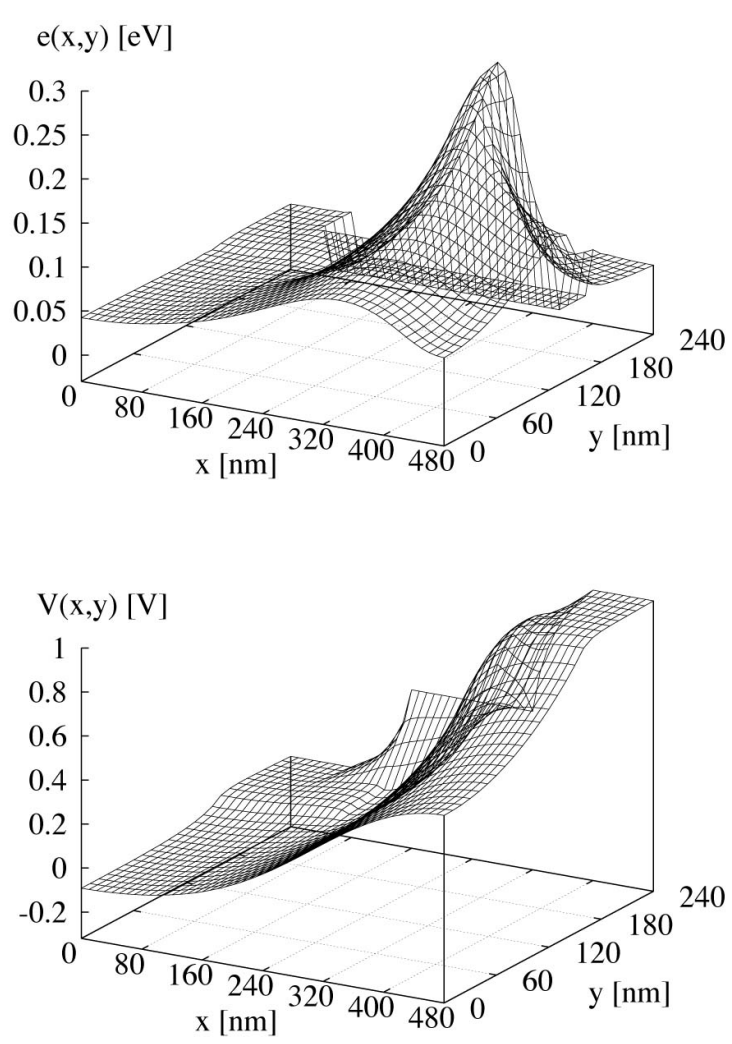

Figure 3. Macroscopic quantities as functions of $x$ and $y$ at $t=5$ ps: electron density (top left); electron energy (top right); velocity field and current lines (bottom left); electric potential (bottom right).

for the $y$-position of the interface. The numerical fluxes $\hat{G}_{i, j_{*}+1 / 2, k, m, n}(t)$ and $\hat{G}_{r i,-1 / 2, k, m, n}^{1}(t)$ are two approximations of the actual flux across the interface at $x_{i}$. In Figure 2 the positions of these fluxes are displayed by the filled triangles. A conservative approximation is obtained, if we use the same flux for determining the derivatives $\partial_{y} G_{i, j_{*}, k, m, n}$ on the coarse and $\partial_{y} G_{r i, 0, k, m, n}^{1}$ on the fine grid.

Concerning the Poisson equation, we use the finite-difference approximation [3] to discretize the differential operator on the left-hand side of $(3)$ on both grids $\mathcal{D}$ and $\mathcal{D}^{1}$. The required quantities $V\left(x_{i}^{1}, y_{0}^{1}-\Delta y / r\right)$ are obtained by a centered linear interpolation in real space in terms of the potential values on the coarse grid.

\section{Numerical Results}

In this paper, we show the results of a two-dimensional simulation of the silicon MOSFET depicted in figure 1. To this end we solve the Boltzmann-Poisson system by applying our new numerical scheme with a weight function $p$ being equal to the function $s(w)$ defined in $(7)$. The donor density $N_{D}$ is chosen as $3 \times 10^{17} \mathrm{~cm}^{-3}$ in the $n^{+}$regions and null in the other part of the device. Holes are completely neglected in this simulation. Hence, the bulk substrate is treated as intrinsic silicon. Source and drain contacts are modeled as ohmic contacts, which allow the electrons to enter and exit the device. Perfectly reflecting boundary conditions are imposed at the $\mathrm{Si} / \mathrm{SiO}_{2}$ interface and at all of the non-contact surfaces of the MOSFET. 
The electrostatic potential is assigned to $V_{S}=0 \times V_{*}$ at source, $V_{G}=0.4 \times V_{*}$ at gate and $V_{D}=1 \times V_{*}$ at drain. Moreover, we assume a vanishing electric field in the direction normal to the surface at the bottom, right and left sides, as well as at the top except for the contact regions. The relative dielectric function $\epsilon_{r}$ holds the value 3.9 in the $\mathrm{SiO}_{2}$ region and 11.7 in the other part of the device.

Since we study a time dependent problem, initial conditions must be fixed. We choose the distribution function $f(0, \mathbf{x}, \mathbf{k})$ to be given by a Maxwellian of zero bulk velocity at the lattice temperature $T_{L}$ and the initial density equal to the donor density.

\subsection{Uniform Grids}

First, we present results obtained with a uniform discretization of the real space variables. The number of grid points in the $(x, y)$ domain is set to $49 \times 25$. Further, we choose $N_{w}=33, N_{\mu}=8, N_{\varphi}=8$ and $\sigma=3$. The plots of figure 3 show the results of the numerical simulation for important macroscopic quantities at $t=5$ ps. After a temporal evolution of 5 ps the stationary state is approximately reached. At the top in figure 3 , we exhibit two main moments of the distribution function, namely the electron density and the averaged energy:

$$
n(\mathrm{t}, \mathbf{x})=\int_{\mathbb{R}^{3}} f(\mathrm{t}, \mathbf{x}, \mathbf{k}) d \mathbf{k}, \quad e(\mathrm{t}, \mathbf{x})=\frac{1}{n(\mathrm{t}, \mathbf{k})} \int_{\mathbb{R}^{3}} f(\mathrm{t}, \mathbf{x}, \mathbf{k}) \varepsilon(\mathbf{k}) d \mathbf{k}
$$

The integrals (20) are evaluated using the midpoint rule. The plots at the bottom of figure 3 show the velocity field and the current lines versus position as well as the electrostatic potential.

We performed comparisons of the relevant hydrodynamical quantities in the stationary regime with the corresponding data obtained by the DSMC method. The overall agreement between the BTE and the DSMC results turned out to be very good. Perfect coincidence of the results was found for the electrostatic potential and the electric field. However, small differences of the electron density and the average velocity occured in isolated regions of the device. To show these typical differences, we plot the cuts of the density and the ycomponent of the mean velocity, $v_{y}$, for $y=120 \mathrm{~nm}$ and $y=200 \mathrm{~nm}$ in figure 4 . In our opinion, the differences arise for two main reasons. First we state the poor accuracy of DSMC results in regions where the charge density is low. In these parts of the device, however, the distribution function is smooth enough so that we can rely on the high quality of the deterministic numerical solution of the Boltzmann-Poisson system. The second origin of the observed differences lies in the difficulty to impose exactly corresponding boundary conditions for the BTE and DSMC models.

\subsection{Non-Uniform Grids}

In the following, we present results of the MOSFET-simulation obtained by applying the non-uniform discretization of the real space described in section 3. For the coarse grid $\mathcal{D}$ we use $N_{x}=24$ and $N_{y}=12$. Since we want to cover the upper half of the device with the fine grid $\mathcal{D}^{1}$, we choose $j_{*}=6$. Further, we fix the refinement factor by setting $r=3$, which leads to $N_{x}^{1}=72$ and $N_{y}^{1}=16$.

At the top in figure 5 we present two-dimensional mesh plots of the particle density and the average electron energy in the stationary state. The $x$-component of the mean electron velocity and the electric potential are displayed in the plots at the bottom of figure 5 . We observe that the considered quantities are perfectly smooth at the interface between the coarse and the fine grid. The plots show that all of the strong gradients of the solution are located in the upper half of the MOSFET. Therefore, the strong variations of the macroscopic quantities are well resolved in the fine grid.

In order to test the accuracy of the results obtained with the non-uniform discretization we perform a detailed comparison with results stemming from a reference calculation on a uniform grid with $N_{x}=72$ and $N_{y}=36$. This uniform discretization provides the high resolution of the fine grid $\mathcal{D}^{1}$ in the entire device. In figure 6 we depict macroscopic quantities as functions of $x$ for different fixed $y$-positions in the stationary state. The solid lines display the results of the reference calculation and the markers present the data obtained with the non-uniform discretization in real space. The comparisons presented in figure 6 demonstrate excellent agreement 

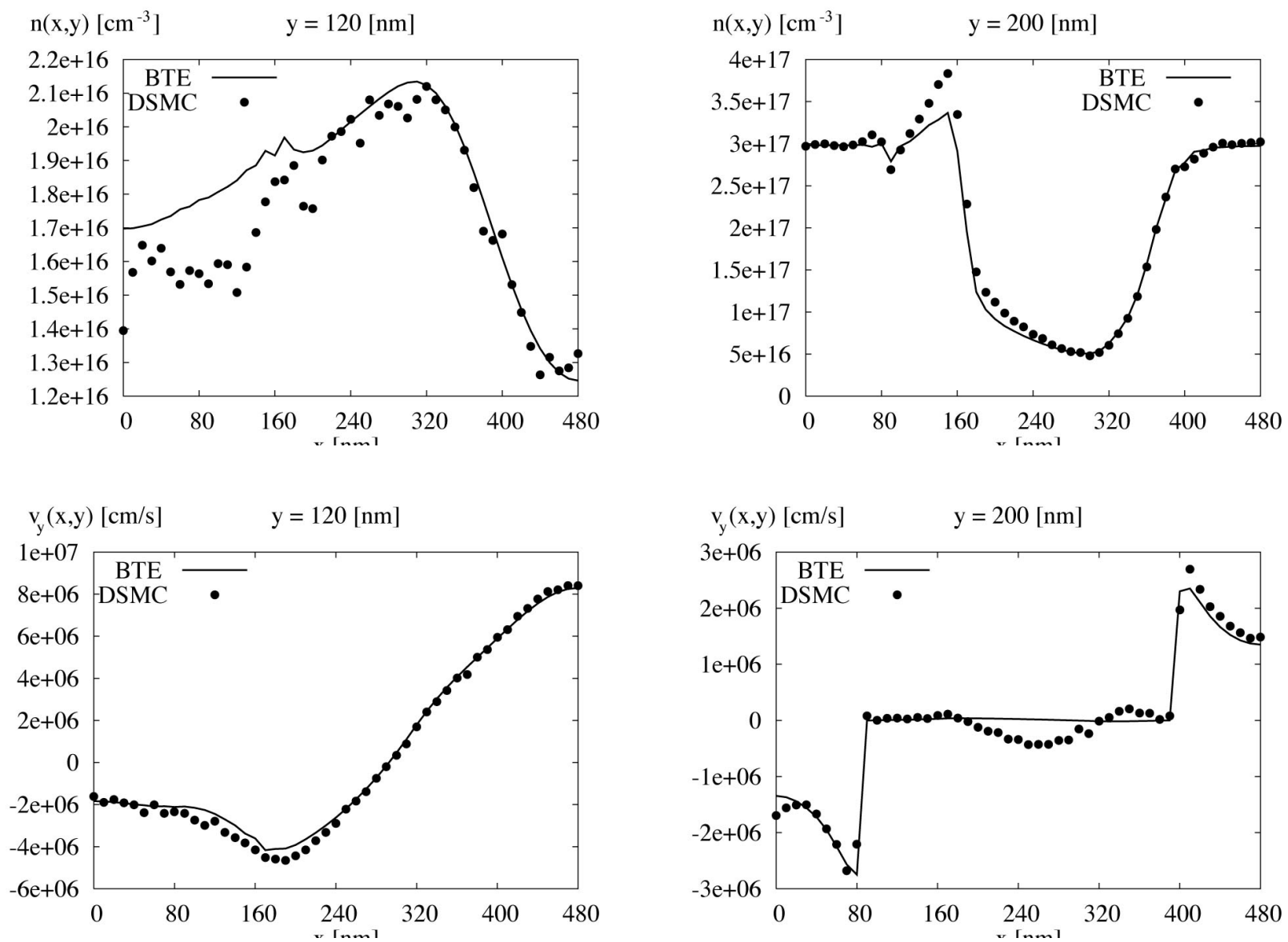

FIgurE 4. Comparison of BTE and DSMC results in the stationary state: electron density as function of $x$ at $y=120 \mathrm{~nm}$ (top left) and $y=200 \mathrm{~nm}$ (top right); y-component of the velocity as function of $x$ at $y=120 \mathrm{~nm}$ (bottom left) and $y=200 \mathrm{~nm}$ (bottom right).

between them. Even at the interface between the fine and the coarse grid, i.e. at $y=120 \mathrm{~nm}$, the correspondence between the results is very good. To perform the simulation in the time interval [0,5] ps, the required CPUtimes are $t_{u} \approx 561$ minutes for the uniform grid and $t_{n u} \approx 282$ minutes in the case of the non-uniform grid. The computations were performed with a AMD Athlon XP 2500+/1822 MHZ processor. This means that the application of a non-uniform discretization in real space reduces the computational effort of the simulation by $100 \%$, while the accuracy of the results remains unaffected. 

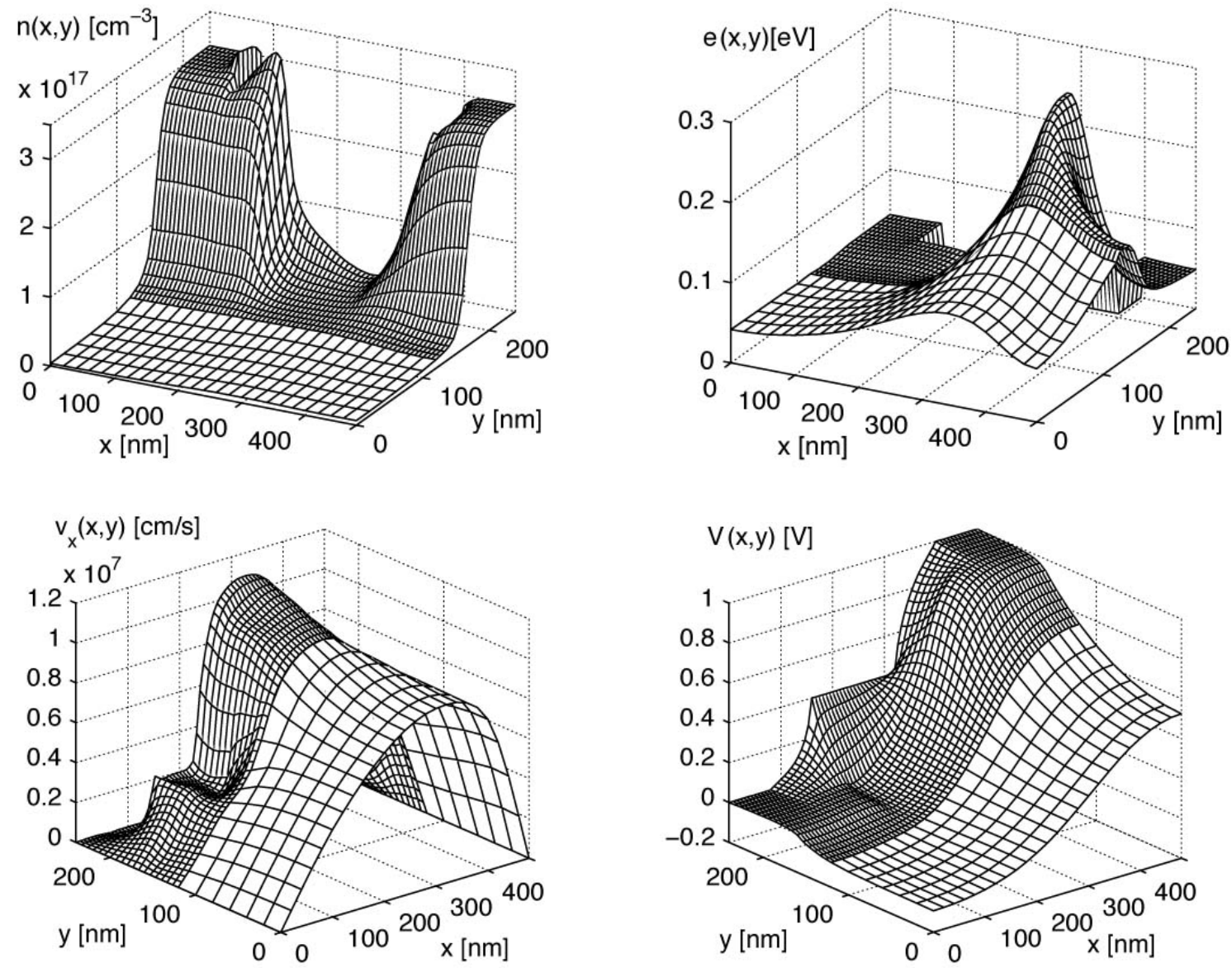

Figure 5. Stationary-state quantities: electron density (top left), mean electron energy (top right), $x$-component of the mean electron velocity (bottom left) and electric potential (bottom right) as functions of $x$ and $y$. 

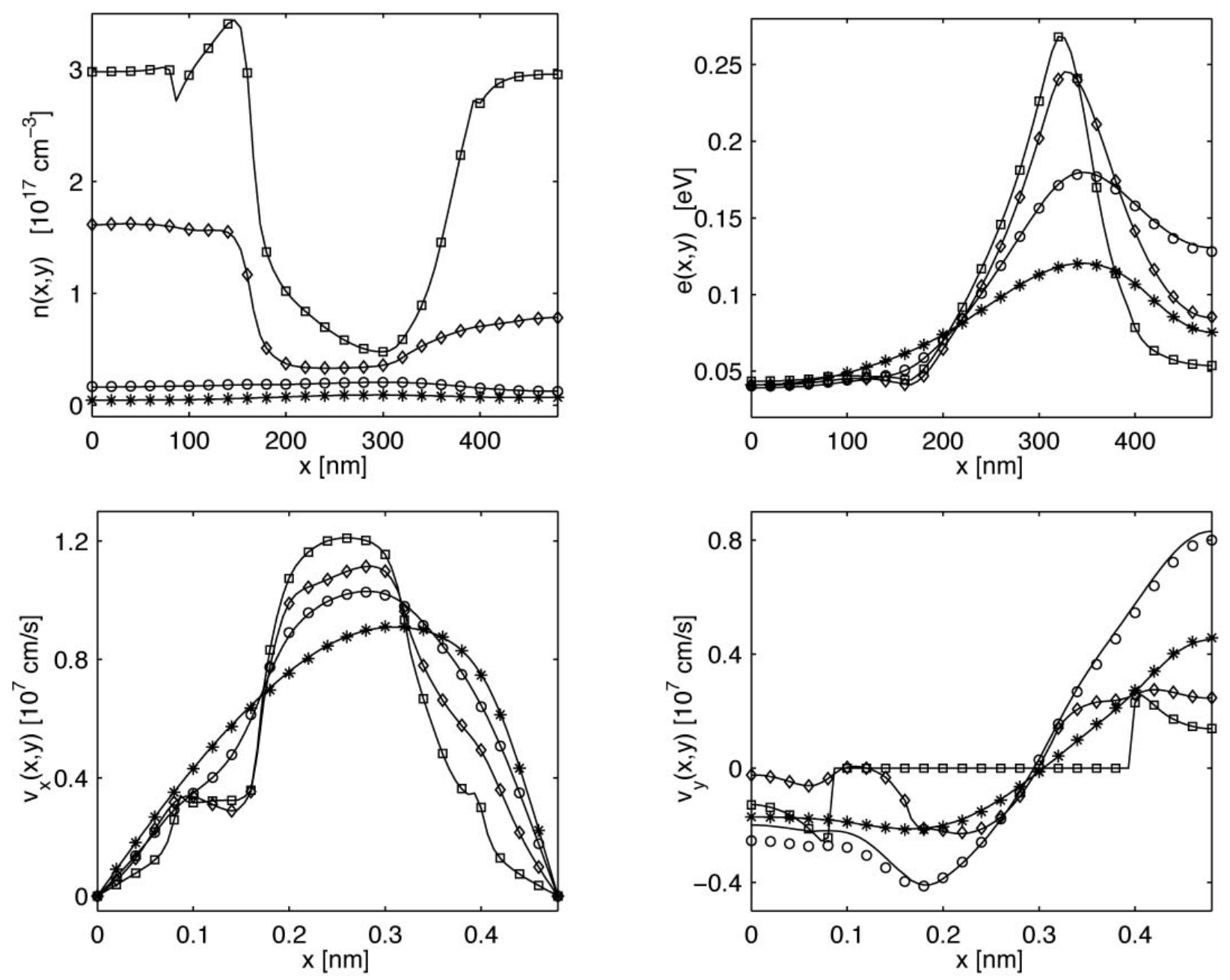

Figure 6. Comparison of macroscopic quantities: electron density (top left), mean electron energy (top right), $x$-component (bottom left) and $y$-component of the mean electron velocity (bottom right) as functions of $x$ for fixed $y$. The markers $\square, \diamond$, $\circ$ and $*$ refer to quantities obtained with the non-uniform grid at $y=40 \mathrm{~nm}, y=120 \mathrm{~nm}, y=160 \mathrm{~nm}$ and $y=200 \mathrm{~nm}$ and solid lines represent results of the reference calculation. 


\section{REFERENCES}

[1] P.A. Markowich, C. Ringhofer, and C. Schmeiser, Semiconductor Equations, Springer-Verlag, New York, 1990.

[2] D.K. Ferry, Semiconductors, Maxwell MacMillian, New York, 1991.

[3] C. Jacoboni and P. Lugli, The Monte Carlo Method for Semiconductor Device Simulation, Springer-Verlag, New York, 1989.

[4] K. Tomizawa, Numerical Simulation of Submicron Semiconductor Devices, Artech House, Boston, 1993.

[5] C. Jacoboni and L. Reggiani, The Monte Carlo method for the solution of charge transport in semiconductor with application to covalent materials, Review Modern Phys. 55 (1983), 645-705.

[6] M.V. Fischetti, Monte Carlo simulation of transport in technologically significant semiconductors of the diamond and zincblende structures - Part I: homogeneous transport, IEEE Trans. Elec. Dev. 38 (1991), 634-649.

[7] J.A. Carrillo, I.M. Gamba, A. Majorana, and C.-W. Shu, A WENO-solver for 1D non-stationary Boltzmann-Poisson system for semiconductor devices, J. Comput. Electr. 1 (2002), 365-375.

[8] J.A. Carrillo, I.M. Gamba, A. Majorana, and C.-W. Shu, A WENO-solver for the transients of Boltzmann-Poisson system for semiconductor devices: performance and comparisons with Monte Carlo methods, J. Comput. Phys. 184 (2003), 498-525.

[9] J.A. Carrillo, I.M. Gamba, A. Majorana, and C.-W. Shu, A direct solver for 2D non-stationary Boltzmann-Poisson systems for semiconductor devices: a MESFET simulation by WENO-Boltzmann schemes, J. Comput. Electr. 2 (2003), 375-380.

[10] M. Lundstrom, Fundamentals of Carrier Transport, Cambridge University Press, Cambridge, 2000.

[11] J.M. Ziman, Electrons and Phonons. The Theory of Transport Phenomena in Solids, Oxford University Press, Oxford, 2000.

[12] A. Majorana and R.M. Pidatella, A finite difference scheme solving the Boltzmann-Poisson system for semiconductor devices, J. Comput. Phys. 174 (2001), 649-668.

[13] M. Galler and A. Majorana, Deterministic and stochastic simulations of electron transport in semiconductors, submitted to Transp. Th. and Stat. Phys., 6th MAFPD, Kyoto, Japan, Sept. 19-23, 2004.

[14] C.-W. Shu and S. Osher, Efficient implementation of essentially non-oscillatory shock capturing schemes, J. Comput. Phys. 77 (1988), 439-471.

[15] M.J. Berger and P. Colella, Local adaptive mesh refinement for shock hydrodynamics, J. Comput. Phys. 82 (1988), 64-84.

[16] R. Keppens, M. Nool, G. Toth and J.P. Goedbloed, Adaptive mesh refinement for conservative systems: multi-dimensional efficiency evaluation, Computer Physics Communications 153 (2003), 317-339.

[17] K. Sebastian and C.-W. Shu, Multidomain WENO finite difference method with interpolation at subdomain interfaces, Journal of Scientific Computing 19 (2003), 405-438.

[18] M. Galler, A. Majorana and F. Schürrer, A Multigroup-WENO solver for the non-stationary Boltzmann-Poisson system for semiconductor devices, Proceedings of the SCEE 2004, Capo d'Orlando, Italy, Sept. 5-9, 2004. 